\title{
Educación en contextos rurales: experiencia de articulación entre la Universidad Nacional de Córdoba y el Movimiento Campesino de Córdoba
}

\author{
Carolina Vélez Funes \\ carolinavelezf@gmail.com \\ (iD) orcid.org/0000-0003-4516-0705 \\ María Martinez \\ martinezcav.maria@ffyh.unc.edu.ar \\ (iD) orcid.org/0000-0002-9353-1024
}

\author{
Carina Sansón \\ carina.andrea.sanson@mi.unc.edu.ar \\ (iD) orcid.org/0000-0002-5440-6829

\section{Pía Fontaine} \\ fontainepia@gmail.com \\ (iD) orcid.org/0000-0002-7225-7134 \\ Universidad Nacional de Córdoba, Argentina.
}

Sujetos y relaciones en extensión universitaria / Intervenciones
RECEPCIÓN: 24/06/20

ACEPTACIÓN FINAL: 10/09/20

\section{Resumen}

Desde el año 2009 al 2015 participamos del Programa Nacional de Voluntariado Universitario en un proyecto de extensión vinculado a la educación rural. El proyecto fue diseñado conjuntamente por docentes, estudiantes y egresados de la Facultad de Filosofía y Humanidades de la Universidad Nacional de Córdoba y por miembros del Movimiento Campesino de Córdoba. Surgió a partir de la demanda de acompañamiento al proyecto educativo secundario elaborada por este Movimiento que consistía en la apertura de escuelas campesinas en parajes rurales del norte de Córdoba. El objetivo de nuestra intervención fue acompañar los procesos de enseñanza en las escuelas. A través de ello, se fueron construyendo dinámicas de intercambio de propuestas metodológicas y conocimientos, lo que permitió visibilizar las complejidades y tensiones en la articulación de una institución educativa universitaria pública con un movimiento social y puso a su vez en cuestión la formación universitaria en un contexto de intervención concreta.

Palabras clave: educación; extensión universitaria; ruralidad; movimientos sociales; campesinado.
Education in rural contexts: experience of articulation between the Universidad Nacional de Córdoba and the Movimiento Campesino de Córdoba

\section{Abstract}

From 2009 to 2015 we participated in the National University's Volunteer Program in an extension project linked to rural education. The project was jointly designed by professors, students and graduates of the Facultad de Filosofía y Humanidades of the Universidad Nacional de Córdoba and by members of the Movimiento Campesino de Córdoba. It arose from the request for accompaniment to the secondary educational project prepared by the Córdoba Peasant Movement, which consisted of the opening of Rural Schools in rural areas in the north of Córdoba. The objective of our intervention was to accompany the teaching processes in schools. Through this, a series of methodology and knowledge exchange was built. This allowed to visualize the complexities and tensions in the articulation of a public university educational institution with a social movement, putting in turn the university training in a context of concrete intervention.

Keywords: education; university extension; rurality; social movements; peasantry.

\author{
Educação em contextos rurais: experiência \\ de articulação entre a Universidade \\ Nacional de Córdoba e o Movimento \\ Camponês de Córdoba
}

\section{Resumo}

Participamos do Programa de Voluntariado da Universidade Nacional, de 2009 a 2015, em um projeto de extensão vinculado à educação rural. O projeto foi elaborado em conjunto por professores, alunos e formados pela Faculdade de Filosofia e Humanidades da UNC e por membros do Movimento Camponês de Córdoba (MCC). Surgiu da solicitação de acompanhamento do projeto educacional secundário elaborado pelo MCC que consistia na abertura de escolas rurais em áreas rurais no norte de Córdoba. $\mathrm{O}$ objetivo de nossa intervenção foi acompanhar os processos de ensino nas escolas. Com isso, construiu-se uma dinâmica de troca de propostas e conhecimentos metodológicos que permitiu visualizar as complexidades e tensões na articulação de uma instituição pública de ensino universitário com um movimento social, questionando, por sua vez, a formação universitária no contexto de intervenção concreta.

Palavras-chave: educação; extensão universitária; ruralidade; movimentos sociais; campesinato.

Para citación de este artículo: Vélez Funes, C.; Martinez, M.; Sansón, C. y Fontaine, P. (2020). Educación en contextos rurales: experiencia de articulación entre la Universidad Nacional de Córdoba y el Movimiento Campesino de Córdoba. +E: Revista de Extensión Universitaria, 10(13), e0015. doi: 10.14409/extension.2020.13.Jul-Dic.e0015 


\section{Introducción}

El relevamiento realizado por el Movimiento Campesino de Córdoba (MCC) en el año 2008 sobre la oferta educativa en la región puso en evidencia la inexistencia o inadecuación de servicios educativos en la zona rural del norte y noroeste de la provincia de Córdoba. Por este motivo, el MCC comenzó a diseñar una propuesta educativa para que pudieran abrirse escuelas secundarias en los parajes rurales. El proyecto propuso la creación de escuelas de gestión pública con modalidad de alternancia que fueran cogestionadas por las comunidades. El objetivo central de la propuesta fue garantizar el acceso a la educación obligatoria para las familias del campo a través de escuelas ubicadas donde esas familias residen y en las que se contextualizaran los contenidos, metodologías y modalidades escolares con lo rural.

El diseño de la propuesta tuvo muchas instancias de trabajo y contó con la participación de múltiples actores sociales. Uno de ellos fue la Universidad Nacional a través del Programa de Espacio Rural de la Secretaría de Extensión Universitaria, que convocó a su vez al Programa de Investigación en Educación Básica y Rural de Jóvenes y Adultos (EBRJA). Desde ese momento, este Programa comenzó a acompañar el desarrollo de la propuesta educativa contribuyendo con el relevamiento de datos estadísticos (Cragnolino et al, 2009) e investigando el marco jurídico-normativo que garantiza el derecho a la educación y regula las condiciones en las que éste se efectiviza tanto en el ámbito urbano como rural. También revisó y analizó detalladamente el repertorio de alternativas institucionales (Bowman et al., 2008) y participó de gestiones realizadas ante el Ministerio de Educación de la provincia de Córdoba.

La negociación con el Ministerio, en el marco de diversas tensiones, dio lugar a acuerdos que posibilitaron la apertura de tres sedes de educación secundaria a distancia para adultos, adultas y jóvenes mayores de edad en tres localidades rurales: La Encrucijada, en el departamento de Río Seco; Los Escalones, en el departamento de Cruz del Eje; y La Libertad, en el departamento de Ischilín en el año 2009.

En este contexto, ese mismo año se presentó ante la Secretaría de Políticas Universitarias el proyecto de Voluntariado Universitario "Fortalecimiento de las Escuelas Campesinas de jóvenes y adultos" del que formamos parte y que tenía como objetivo proponer respuestas para las necesidades educativas identificadas. Recuperamos como antecedente del relato de estos procesos el trabajo realizado por miembros del equipo de investigación en Educación Rural y Básica de Jóvenes y Adultos (Cragnolino et al., 2010), del cual se desprende el proyecto de extensión mencionado. Teniendo esto en cuenta, nos proponemos retomar y continuar el relato desde nuestra condición de estudiantes y graduadas extensionistas, compartiendo los conocimientos construidos durante el trabajo efectuado con los docentes de las escuelas para mejorar las intervenciones pedagógicas y didácticas.

El proyecto respondía a una demanda específica de intervención planteada por el MCC: que desde el ámbito universitario pudieran desarrollarse estrategias para acompañar los procesos de formación y sostenimiento de las dinámicas de enseñanza y aprendizaje de las Escuelas Campesinas. La propuesta se construyó sobre los supuestos de que las y los estudiantes y graduados podíamos aportar conocimientos y habilidades para contribuir al desarrollo de los procesos de la escuela y de que los ámbitos de formación para las y los estudiantes universitarios son amplios y diversos, por lo que la experiencia de trabajo con organizaciones nos permitiría adquirir recursos y aprendizajes nuevos. 
Dentro de las distintas perspectivas sobre cómo entender la extensión universitaria y llevar adelante proyectos de este tipo, el posicionamiento ético-político de nuestra práctica extensionista se centró en la reivindicación de derechos básicos y de participación ciudadana, considerando al otro — con quien se trabaja - como sujeto de derecho. Entendemos, junto a autores como Pacheco (2004) y Tommasino y Cano (2016), que la extensión es un espacio de co-construcción con diferentes sectores sociales que supone dinámicas de interacción cooperativa en las que quienes articulan la demanda a partir de la problematización de su propia realidad son protagonistas del proceso.

Desde nuestra perspectiva, la práctica extensionista pone en relación a quienes se ven directamente afectados por una problemática social, en este caso educativa, y a agentes externos a la misma. Y es a través de la construcción compartida de conocimiento que se abordan y definen actividades orientadas al logro de objetivos acordados, teniendo en cuenta, a su vez, las tensiones que atraviesan estos procesos (Achilli, 2017). Desde estos supuestos escribimos con la convicción de que es necesario continuar generando espacios de encuentro entre la universidad pública y los distintos movimientos sociales si queremos favorecer el intercambio entre saberes producidos desde el ámbito académico y saberes construidos a partir de las prácticas colectivas de las organizaciones en los territorios.

Con esta premisa, leímos la demanda de la organización, que consecuentemente se constituyó en un eje central al momento de diseñar la propuesta de extensión. A partir de esta demanda, los equipos conformados por estudiantes, docentes y egresados de distintas carreras establecimos las actividades a realizar: registro y sistematización de clases, revisión de contenidos disciplinares y diseño de talleres de capacitación considerando las necesidades de las y los docentes de las escuelas.

En este escrito presentamos algunos aspectos de esta experiencia de articulación entre una institución universitaria y un movimiento social haciendo referencia no solo a las metodologías de intervención utilizadas, sino también a las dificultades y conflictos surgidos entre los diferentes actores. Otro aspecto sobre el que nos detenemos son las representaciones construidas en torno a la posición que tanto la universidad como el movimiento ocupan en el espacio social y en cuanto a cómo los conocimientos y saberes que detentan se ponen en juego en la situación educativa. Por último, quisiéramos presentar las reflexiones surgidas de esta experiencia ya que creemos que las prácticas de extensión deben ser sistematizadas y compartidas para acumular aprendizajes como comunidad universitaria en pos de nuevas y mejores articulaciones.

\section{El Movimiento y su demanda de acompañamiento a la Universidad}

El MCC es una organización provincial que desde hace aproximadamente 20 años nuclea a familias campesinas que viven en el norte y noroeste de Córdoba. Su objetivo político consiste en articular y aunar esfuerzos para lograr el reconocimiento y acceso a derechos básicos y en mejorar las condiciones de existencia de las familias que participan directa e indirectamente de proyectos y beneficios que la organización promueve en las distintas comunidades. Para esto, las y los militantes del MCC se distribuyen en distintas áreas de trabajo: Territorio, Formación y Educación, Salud Comunitaria, Producción y Comercialización y Jóvenes. 
Asimismo, el MCC forma parte del Movimiento Nacional Campesino Indígena (MNCl); a nivel regional, de la Coordinadora Latinoamericana de Organizaciones del Campo (CLOC); y a nivel mundial, de la Vía Campesina. Y hereda aprendizajes de lucha y organización de movimientos campesinos con mayor trayectoria, como el Movimento dos Trabalhadores sem Terra de Brasil.

Como se mencionó, el diagnóstico realizado sobre la situación educativa en la zona de influencia del MCC evidenció la necesidad de generar nuevas alternativas frente a las ofertas educativas ya existentes en la región. Los altos niveles de repitencia y sobreedad ${ }^{1}$ indicaban que la cobertura educativa mediante las opciones institucionales vigentes era deficiente. A esto se sumaba que las instituciones escolares en estas zonas no contemplaban trayectos de terminalidad educativa para jóvenes y adultos y que el número de escuelas secundarias en parajes rurales era prácticamente inexistente (Cragnolino et al., 2010). La organización señalaba que los contenidos y materiales de las escuelas cercanas estaban dirigidos a un alumno "universal" no campesino; por lo que se propuso un modelo de escuela pública cogestionada por las familias de las comunidades, donde participaran orientadores comunitarios y colectivos pedagógicos que planificaran contenidos y estrategias según las necesidades "productivas, laborales, culturales y organizativas de las comunidades", tal como se plantea en el Documento Propuesta de Escuela Campesina (MCC y SEU, 2008).

En este marco, el Movimiento elaboró el proyecto de Escuelas Campesinas en diálogo y colaboración con docentes de la Escuela de Trabajo Social y con investigadores del Programa de Educación Rural y de Jóvenes y Adultos de la Facultad de Filosofía y Humanidades de la Universidad Nacional de Córdoba.

El proyecto incluyó una propuesta de lineamientos curriculares fundada en principios tales como educación para y por el trabajo y contenidos contextualizados y socialmente útiles, teniendo en cuenta las características particulares de la región y de sus habitantes.

El resultado de la negociación con el Ministerio de Educación de la provincia fue la apertura de tres sedes del Programa de Educación a Distancia dependientes de distintos Centros Educativos de Nivel Medio de Adultos (CENMA) en parajes rurales, que el MCC denomina Escuelas Campesinas. Estas se encuentran vinculadas a las siguientes organizaciones zonales:

- Asociación de Productores del Noroeste de Córdoba (APENOC), que nuclea comunidades de los departamentos Cruz del Eje y Minas.

- Organización de Campesinos Unidos del Norte de Córdoba (OCUNC), de los departamentos de Tulumba, Ischilín y Sobremonte.

- Unión de Campesinos del Noreste de Córdoba (UCAN), de los departamentos de Tulumba y Río Seco.

Cabe destacar que los programas garantizan la terminalidad educativa secundaria para jóvenes y adultos con titulación oficial, con un formato curricular modular y no anualizado, es decir, flexible con relación a la asistencia y a la continuidad de las trayectorias de las y los estudiantes (Martínez, 2013). Además, tienen un formato institucional basado en convenios con organizaciones, lo cual permitió al MCC acordar los términos de implementación

1) Los datos señalados pueden ser consultados en el Proyecto Escuela Campesina Secundaria. 
e incluso elaborar los contenidos y materiales de una novedosa orientación en Agroambiente, que está vigente.

Vemos entonces cómo quedan delineados tres actores colectivos en el marco de la experiencia. Dos de ellos son: la Universidad, mediante el equipo de extensionistas, y el MCC a través de las y los militantes que estaban coordinando el trabajo en las Escuelas Campesinas. El tercer actor, también central, es el Ministerio de Educación de la provincia, presente a través de funcionarios, supervisores, la dirección del CENMA, y las reglamentaciones que marcan los límites de aquello a negociar.

Cabe destacar que muchos y muchas de los coordinadores, cordinadoras y docentes de las escuelas eran militantes del MCC. Este fue un aspecto clave ya que permitía a los equipos de las escuelas ir tomando decisiones respecto de los tiempos, las dinámicas de desarrollo, evaluación de los contenidos, el enfoque y otros aspectos, según las necesidades de sus estudiantes y de la organización. Además de considerar las características del espacio rural y las trayectorias educativas de los estudiantes, el MCC definió una serie de principios para las Escuelas vinculados al conocimiento y a la educación que fueron tomando formas concretas en prácticas y discursos al implementarse el proyecto. Algunos de ellos, ya mencionados, fueron la educación en el territorio y de alternancia y otros, ahora señalados, la educación para la cooperación y la organización y la educación para y por el trabajo.

Uno de los principales desafíos asumidos por el MCC fue revisar los contenidos propuestos por los módulos - libros de texto- ${ }^{2}$ ya que estos habían sido pensados para estudiantes provenientes de contextos urbanos y no consideraban aspectos culturales ni ambientales de la vida campesina, como tampoco integraban la experiencia comunitaria y organizativa. Es por esto que una de las principales tensiones estructurantes que atravesó a la escuela fue y es hasta la fecha la exigencia de cumplir con requisitos y disposiciones ministeriales que permitieran la acreditación del trayecto pero disputando el sentido de lo educativo desde la perspectiva de la organización.

Con el impulso de las organizaciones zonales de las que dependían, las escuelas iniciaron un proceso que, a pesar de encontrarse con obstáculos, logró un desarrollo exitoso a través de dinámicas propias y prácticas de organización. En el marco de los ritmos de implementación de la propuesta en cada sede, acordamos con los equipos de formación de las escuelas en orientar el proyecto de voluntariado universitario a las siguientes tareas: sistematizar el trabajo de las escuelas, jerarquizar contenidos, guiar a los equipos docentes y avanzar en el diseño de una contextualización curricular para las Escuelas Campesinas. La incorporación de la Universidad con su fuerte capital simbólico en estos procesos precisó de diálogos y consensos con los equipos docentes del MCC que coordinaban cada una de las escuelas. Si bien en la negociación con el Ministerio de Educación la institución universitaria aparece como aliada al MCC, debemos destacar que ninguno de los tres sujetos colectivos intervinientes en la apertura de las escuelas puede ser considerado homogéneo ya que al interior de ellos conviven diferentes perspectivas y miradas sobre los procesos presentados, lo cual evidencia la complejidad en la construcción de acuerdos. 
En un comienzo se realizaron encuentros de planificación donde los docentes y estudiantes miembros del voluntariado se reunían con los equipos de formación de las distintas organizaciones zonales para coordinar talleres y actividades solicitadas por el MCC en función de los temas propuestos por docentes y por los alumnos de la Escuela Campesina. La construcción y formulación de esa demanda en el vínculo extensionista fue modificándose de manera dinámica y se dio en el marco del inicio de las actividades escolares.

En las reuniones entre las distintas sedes y los extensionistas, la negociación por la distribución de recursos económicos, temporales y humanos, generaba tensiones permanentes. Como cada escuela dependía de una organización zonal distinta (APENOC, OCUNC y UCAN respectivamente), cada interlocución arrastraba definiciones previas de dichas organizaciones con relación a las condiciones de implementación y de intervención de la Universidad. Las representaciones sociales de los coordinadores y docentes de las escuelas sobre las condiciones, tiempos y modos académicos fueron atravesando nuestra práctica extensionista, como también lo hicieron nuestras prenociones, en algunos casos románticas, sobre el trabajo territorial del movimiento y la vida en el campo.

A esto se sumaban nuestros posicionamientos ético-políticos y nuestras construcciones personales y colectivas sobre el conocimiento y el lugar de la extensión en todo ese proceso. Estas miradas sobre la realidad del campo y del Movimiento, sobre la Universidad, la educación y los aportes de extensionistas y militantes en esta instancia de trabajo compartido fueron moldeando las vinculaciones entre los sujetos intervinientes. $Y$ en ese proceso de des/ encuentros e intercambios se fueron configurando y reconfigurando los imaginarios acerca de quién era el otro y del espacio compartido.

Por otra parte, la recepción de la propuesta en las distintas sedes no fue la misma. En algunos casos se generó más afinidad con algunos sujetos que con otros no solo en lo vincular sino también en cuanto a la fluidez de la dinámica de trabajo. En eso además influyeron fuertemente las condiciones materiales objetivas de cada sede y escuela (la distancia, el espacio físico, la cantidad de docentes y militantes, el transporte, etc.) y la manera en que tanto docentes como estudiantes construyeron la cotidianeidad de la experiencia educativa. En el caso de la Escuela Campesina ubicada en el paraje Los Escalones —que pudo resolver ciertas dificultades de organización y funcionamiento y que además contaba con un grupo nutrido de estudiantes- se promovió con mayor rapidez y frecuencia la participación de las y los extensionistas en la escuela, lo cual posibilitó una frecuencia de intercambios más constante.

\section{Actividades de extensión en las escuelas: tensiones y estrategias}

Mediante reuniones de planificación y evaluación con los equipos docentes de las escuelas se fue articulando el trabajo conjunto y la construcción de dinámicas que permitían la convergencia e intercambio de conocimientos y propuestas metodológicas.

Las actividades más relevantes llevadas a cabo durante la práctica de extensión fueron: la planificación de acciones junto con los docentes de las escuelas y al interior de los equipos extensionistas; las visitas a las distintas escuelas campesinas; la recopilación, producción y sistematización de registros e información; la revisión de los módulos provistos por el Ministerio para la adaptación de sus temáticas a las necesidades de las Escuelas Campesinas; la 
elaboración e implementación de talleres de capacitación tanto para los equipos docentes como para los alumnos de las escuelas y la producción de documentos incluyendo los contenidos, metodologías y estrategias de trabajo pensadas para cada taller con el fin de que pudieran ser compartidos y reutilizados.

En un primer momento, nos organizamos en comisiones de manera similar a la de los equipos de formación de las escuelas. Esta respondía a la distribución del tiempo escolar y a la organización de contenidos en las áreas de estudio propuestas por el programa de terminalidad: Lengua, Matemática, Ciencias Sociales y Ciencias Naturales. Así, según la formación académica que teníamos y con intención de poner en juego el conocimiento adquirido en los equipos de investigación, docentes, estudiantes y egresados de las carreras de Letras, Filosofía, Ciencias de la Educación, Matemática, Historia, Geografía, Antropología y Geología, nos agrupamos en esas áreas temáticas.

Al interior del grupo extensionista, la forma de trabajo se fue transformando conforme a las necesidades y demandas de la escuela y a las posibilidades de los participantes. Destacamos, como mencionan Cragnolino et al. (2010), que se pudo construir un equipo de trabajo interdisciplinar que produjo colectivamente a partir de la trayectoria y especificidad académica de cada uno de sus miembros. Y que se logró vincular y repensar saberes teórico-académicos con conocimientos de las comunidades campesinas y otros que surgieron de la intervención en las escuelas.

Es importante resaltar igualmente que el desarrollo del proyecto fue condicionado por la disponibilidad de tiempo y las posibilidades individuales para sostener el espacio. Fue complejo coincidir en horarios y actividades y acordar ideas y dinámicas. Sumado a esto, el grupo de trabajo no se mantuvo estable, sino que hubo rotación e incorporación de nuevos participantes en función de las cambiantes necesidades del proyecto.

Por otra parte, estas mismas dificultades para coordinar actividades al interior del equipo extensionista se presentaron al intentar programar encuentros y actividades con coordinadores y docentes de las escuelas. A las complejidades propias de los tiempos acotados y de las urgencias de la organización, se agregaron la distancia y el costo económico de los traslados, ya que los parajes en donde están las escuelas se encuentran aproximadamente a 200 kilómetros de Córdoba Capital.

Si bien cada área de trabajo contaba con conocimientos sólidos sobre los saberes disciplinares correspondientes para poder acompañar los procesos educativos de las escuelas, también era necesario conocer más sobre la realidad campesina y sobre los estudiantes. Por ello, era preciso entrar en contacto poco a poco y respetuosamente con las prácticas y dinámicas escolares propias de un espacio social que no nos era familiar y ponerlas en relación con otras dimensiones de la realidad: políticas, económicas e históricas, como propone la etnografía analítica (Cragnolino, 2017).

Por ello, los equipos de formación nos propusieron viajar al campo para que en esos viajes pudiéramos conocer las escuelas. Esto nos permitió tomar contacto no solo con los estudiantes sino también con los equipos docentes y las condiciones materiales de las escuelas, y de ese modo comprender mejor cuáles eran los límites y posibilidades para aprender y enseñar. Durante estos viajes participamos de las actividades escolares que registramos y además fuimos invitadas a distintos eventos sociales y culturales. Compartir estos espacios nos permitió generar nuevos vínculos y aproximarnos a lo campesino desde otros lugares. 


\section{El registro}

Desde el inicio del proceso, el registro se consideró una herramienta fundamental para diseñar los talleres de capacitación y producir documentos. Resultó un aporte importante para comprender qué sucedía en la escuela ya que nos posibilitó acceder a los procesos de construcción de la cotidianeidad que involucraba a docentes y estudiantes (Achilli, 2017).

Para llevar adelante esta tarea, se entregó a cada escuela un grabador para registrar las clases. Los equipos de formación de las escuelas decidieron ser quienes las desgrabaran. De esta tarea también participaron en algunas ocasiones estudiantes universitarios de la carrera de Trabajo Social que allí llevaban a cabo sus prácticas profesionales.

En el registro quedó asentado el desarrollo de las clases observadas: los recortes de contenidos, los distintos recursos técnicos y pedagógicos, y las dificultades o aspectos sin respuestas que indicaban la necesidad de intervención. A su vez, nos dio pistas sobre la manera en que se posicionaban los docentes frente al conocimiento y cómo entendían la relación enseñanza-aprendizaje.

Estos procesos de registro, desgrabación y posterior sistematización se llevaron a cabo con lentitud debido a la complejidad que cada instancia planteaba. Como sabemos, el trabajo de observación y registro manifiesta la impronta subjetiva de quien lo realiza, aunque se establezcan pautas generales para el desarrollo de la actividad, lo cual es un elemento a tomar en cuenta a la hora de analizarlos. A su vez, la desgrabación puede verse obstaculizada por desperfectos técnicos en los equipos y/o condiciones desfavorables del contexto de grabación, lo que puede entorpecer el proceso de análisis.

Para efectuar la sistematización de los registros disponibles no solo utilizamos los producidos por nosotros sino también los realizados por docentes de las escuelas. Cada área definió las categorías que le parecían relevantes para organizar la información, teniendo en cuenta las demandas de las escuelas y los ejes solicitados por los equipos de formación, ya que el resultado de ese trabajo serviría para planificaciones posteriores y el armado de nuevos materiales.

La riqueza de esta tarea quedó evidenciada en la multiplicidad de niveles de análisis que ofreció. Por un lado, permitió comprender aspectos de la realidad campesina y del proceso de lucha por el territorio llevado a cabo en las comunidades. En el plano institucional, contribuyó a identificar las prácticas sociales y educativas que tienen lugar en la escuela: el desarrollo de la jornada escolar, la manera en que se la divide temática y temporalmente, las actividades que se realizan y aspectos particulares de cada escuela.

Desde lo pedagógico, posibilitó reconocer distintas estrategias para abordar los contenidos, disparidad y diversidad de trayectos pedagógicos con los que los alumnos llegan a la escuela, tensiones y dificultades para retomar temáticas trabajadas en clases anteriores, estrategias didácticas exitosas que convenía sostener, etcétera.

Son varios los motivos por los cuales destacamos la importancia de los registros y las sistematizaciones. En primer lugar, se aprende de la propia práctica. En segundo lugar, estos instrumentos no solo nos permiten revisar, cuestionar y reelaborar las prácticas específicas de la escuela sino también, a partir de ellas, realizar adecuaciones necesarias de los contenidos de los módulos para poder responder a las particularidades de la realidad campesina. En tercer lugar, facilitan la socialización de la experiencia con las otras escuelas del MCC, aportan preguntas y arriesgan respuestas que enriquecen la singularidad con la que cada una construye y transita la experiencia educativa. 
Y por último, si consideramos a la educación rural desde una perspectiva más general, el conocimiento producido en las escuelas campesinas, aun siendo un proyecto con características particulares, puede contribuir a la formación de docentes rurales y puede ser recuperado para pensar otras experiencias en educación rural y de jóvenes y adultos.

\section{La revisión de los módulos}

La revisión de los materiales propuestos por el Ministerio tenía como objetivo analizar los contenidos que allí se presentaban y proponer una secuencia didáctica para su enseñanza. A partir de la lectura del material, la discusión sobre su organización y presentación, y del conocimiento sobre la forma de trabajo de cada escuela, describimos los ejes temáticos presentes y evaluamos en qué casos la propuesta necesitaba ser contextualizada y adaptada. Propusimos, a su vez, recursos pedagógicos diversos y abordajes metodológicos que consideramos útiles para favorecer la aprehensión de los contenidos propuestos.

Teniendo en cuenta el carácter procesual y relacional del aprendizaje, nos pareció importante definir categorías teóricas que pudieran retomarse a través de los distintos módulos, contribuir a integrar y profundizar conocimientos previos y permitir el encadenamiento con los temas anteriores y posteriores. Este esfuerzo por secuenciar temas y contenidos teóricos proponiendo niveles de abordaje progresivos fue muy valorado por los equipos docentes de las Escuelas Campesinas. Ellos ya habían definido claramente cuáles eran los contenidos prioritarios para la escuela, pero en muchos casos necesitaban orientación y asistencia para diseñar dispositivos que permitieran su comprensión. Algo sumamente significativo si tenemos en cuenta que la mayoría de los estudiantes contaba con trayectos escolares frágiles.

Por otra parte, para el equipo extensionista fue de fundamental importancia que los y las docentes de las escuelas aportaran información sobre las trayectorias de los y las estudiantes y las condiciones sociales, ambientales y políticas de la región para que los ajustes de contenidos efectivamente respondieran a la intencionalidad de contextualización. Nos orientaron también en la revisión y el diseño de las estrategias metodológicas utilizadas para presentar temas y actividades a través de sugerencias tales como el uso de materiales visuales, explicaciones cortas y precisas, preguntas que posibilitaran recuperar saberes previos y la elaboración de escritos a partir de situaciones conocidas.

El trabajo documental realizado se centró también en establecer relaciones con acontecimientos del ámbito provincial o nacional y con problemáticas locales. $Y$ en hacer una referencia permanente al espacio social rural, a las condiciones de vida de sus pobladores, a sus características identitarias, a sus actividades productivas, etc. Esta tarea de análisis y reflexión generó discusiones interesantes al interior de las comisiones, donde los aportes de cada disciplina permitieron enriquecer la propuesta e intercambiar las experiencias de los estudiantes en las distintas escuelas.

\section{Los talleres}

Los registros sistematizados y las revisiones de contenidos fueron insumos primordiales para la realización de los talleres porque aportaron información valiosa para pensar cómo diseñarlos e implementarlos, ya que pudimos identificar temas de interés, ritmos de trabajo y 
dificultades a la hora de resolver consignas. Los talleres nos permitieron articular distintos saberes con las demandas alrededor de las cuales fueron construidas nuestras intervenciones. Partimos de preguntas que nos interpelaban para pensar qué relevancia podía tener para los estudiantes aprender determinados conocimientos; fuimos articulando, poniendo en cuestión y jerarquizando saberes académicos para poder desarrollar contenidos integradores.

Los primeros talleres estuvieron dirigidos a los docentes de las escuelas con el objetivo de revisar secuencias didácticas y mostrar algunas estrategias metodológicas. Pero pronto nos solicitaron talleres para los estudiantes donde fuera factible observar in situ la manera en que se podían comunicar/transmitir los conocimientos a los estudiantes.

Para evaluar esas experiencias, pedimos a los docentes que registraran las clases y realizaran devoluciones que pudiésemos incorporar en la definición de temas y actividades para los talleres siguientes. Las preguntas que les propusimos para estructurar las evaluaciones se centraron en la relación entre los contenidos dictados, los conocimientos previos y los nuevos, los vínculos posibles con las demás asignaturas y con los objetivos generales de la escuela en cuanto al concepto de "educación campesina".

Todas las actividades que realizamos durante la práctica extensionista se caracterizaron por procesos de retroalimentación con las y los docentes a través de intercambios y evaluaciones que no solo permitieron adecuar las propuestas ofrecidas a las necesidades educativas de la escuela sino también producir conocimiento nuevo a partir de imbricar el saber académico con el saber de la práctica docente y el saber campesino (Tomassino y Cano, 2016).

\section{Consideraciones finales}

Entendemos que los proyectos de extensión pueden constituirse en instancias dinámicas de problematización y aprendizaje. A través de ellos, los y las estudiantes universitarios se integran a la sociedad en sus distintos ámbitos y realidades, responden a sus demandas al participar en la concreción de su desarrollo y además se preparan como profesionales. De este modo, el papel de la extensión es fundamental en la formación universitaria ya que crea conciencia ciudadana y fortalece la formación en la diversidad. En consecuencia, entendemos que el tránsito por la universidad no es solo para el individuo que tiene la posibilidad de educarse en ella, sino que es un espacio de formación y construcción del tejido social.

El proyecto de extensión "Fortalecimiento de las Escuelas Campesinas de adultos" vincula a la Universidad con una organización social. Esta tiene una trayectoria, un recorrido, una historia de trabajo y lucha. Además, una posición claramente definida respecto de la actuación del Estado en la educación. Desde la perspectiva de la organización, no se concibe la educación como práctica unilateral, al Estado como agente educador, sino que se busca una educación pública de cogestión donde el papel de las familias y del saber rural es central. A partir de las consideraciones presentadas en este artículo, nos parece relevante señalar el lugar central que tiene para los movimientos sociales el diseño de propuestas educativas alternativas, ya que a las exigencias de distribución del poder económico y de participación política se suma la disputa por la construcción de políticas públicas en educación (Vélez Funes, 2020).

Dentro del ámbito educativo, este voluntariado universitario se abre a una nueva realidad, un sector de la sociedad que genera otras dinámicas y pone en cuestión propuestas educa- 
tivas oficiales. Se vuelve igualmente un desafío que admite socializar formas de concebir la educación y distintas miradas sobre la realidad, desafío que lleva a una construcción a través del intercambio y el análisis permanente.

El acercamiento de miembros de la comunidad universitaria a los parajes rurales del norte de Córdoba significa un encuentro con una realidad diferente: la campesina, que interpela a las y los estudiantes y a su formación académica, y al mismo tiempo a la Universidad con relación a los procesos de formación profesional que en ella se ofrecen.

Esta experiencia se trata del diálogo entre la Universidad y el MCC: entre estudiantes y egresados extensionistas, alumnos de las Escuelas Campesinas, coordinadores pedagógicos, docentes de las escuelas, y docentes universitarios que guían el voluntariado. Este diálogo implica reconocer, confrontar, aunar y flexibilizar intereses; genera un intercambio de visiones y posiciones respecto del trabajo que se va llevando a cabo de manera conjunta. $Y$ en esos procesos, tal como señala Achilli (2017), la interacción social entre extensionistas y los integrantes de las escuelas es "dialécticamente modificante". Así, el tránsito de este proyecto posibilita múltiples aprendizajes sobre el territorio, los sujetos y las prácticas socioeducativas, como también la identificación de los significados que estos le atribuyen a su realidad de vida. A su vez, muchos aspectos de esta experiencia se tradujeron en preocupaciones teórico-analíticas que abrieron interesantes procesos de investigación en torno a procesos sociales y educativos en el ámbito rural (Fontaine, 2018; Martínez, 2013; Sansón, 2015; Vélez Funes, 2020).

Como se mencionó, el MCC no circunscribe sus iniciativas y acciones al ámbito educativo, sino que sus demandas abarcan otros derechos básicos que no son garantizados por el Estado. Por tal motivo, los docentes miembros de la organización se encuentran afectados a diversas tareas simultáneamente, y esto incide en las actividades educativas que se desarrollan. Para nosotros fue un aprendizaje importante respetar los tiempos de trabajo de la organización y reconocer que lo que genera la intervención y la sostiene es la demanda.

Las actividades planificadas respondieron a los acuerdos que se realizaron cuando tuvimos oportunidad de encontrarnos. Esto también supuso dinámicas particulares puesto que las sedes a las que pertenecen las escuelas tenían necesidades y características propias. En algunas ocasiones, la urgencia por resolver aspectos productivos, de salud o territoriales, impedía o retrasaba la posibilidad de encuentros o talleres acordados.

Para concluir, podemos decir que valoramos enormemente esta experiencia formativa en dos sentidos. Por un lado, consideramos que los documentos escritos: registros, sistematizaciones, informes y revisión de módulos, son un aporte para las Escuelas Campesinas y otras experiencias educativas que queda a disposición para ser utilizado a corto, mediano o largo plazo, teniendo en cuenta asimismo que la experiencia formó parte de un trabajo más amplio de acompañamiento y fortalecimiento, ya que los procesos de organización y consolidación de las escuelas fueron prolongados. Por otro lado, reafirmamos la importancia crucial de la práctica extensionista en la formación de todo estudiante universitario. Es una instancia en la que se ponen en juego los aprendizajes adquiridos y se alcanzan, mediante otras prácticas y diversos contextos, conocimientos y enfoques nuevos. Se entra en contacto con distintos hechos, objetos, personas y organizaciones transformando así las dinámicas de inserción y además se abre un espacio oportuno para que las y los estudiantes realicen un proceso de evaluación y reflexión sobre la propia formación. 
Por todo esto, consideramos que la extensión es significativa en múltiples sentidos: como herramienta de formación para el estudiante, como vía de acceso y contacto de la universidad con la sociedad, y como instancia legítima de producción de conocimiento y transformación de la realidad.

\section{Referencias bibliográficas}

Achilli, E. (2017). Construcción de conocimientos antropológicos y coinvestigación etnográfica. Cuadernos de Antropología Social, (45). Universidad Nacional de Rosario.

Cragnolino, E. (2017). Desde las escuelas primarias a las escuelas secundarias campesinas. Luchas por la educación pública en Córdoba, Argentina. Educação \& Sociedade, 38(140), 671-688. http://dx.doi.org/10.1590/ es0101-73302017177882

Cragnolino, E.; Del Prato, M. F.; Brumat, M. R.; Caciorgna, L.; Ominetti, L. y Piccioni, A. (2010). Desde la investigación a la extensión, en la construcción colectiva de políticas públicas en educación rural. E+E: Estudios de Extensión en Humanidades, (2). Universidad Nacional de Córdoba.

Fontaine, M. P. (2018). Discurso político y conformación identitaria. Utopías del presente. Apenoc - MCC. Trabajo Final de Licenciatura. Facultad de Filosofía y Humanidades. Universidad Nacional de Córdoba.

Martínez, M. (2013). El para quién del Estado Educador: un contraste entre políticas de Estado y aportes de una organización social a una propuesta pedagógica para jóvenes y adultos trabajadores. Trabajo Final de Licenciatura. Facultad de Filosofía y Humanidades. Universidad Nacional de Córdoba.

Pacheco, M. (2004). Reflexiones en torno a la construcción del espacio de la extensión universitaria hoy. Cuadernos de Educación, (3).

Sansón, C. (2015). Prácticas de literatura oral en contextos campesinos del noroeste de Córdoba. Trabajo Final de Licenciatura. Facultad de Filosofía y Humanidades. Universidad Nacional de Córdoba.

Tommasino, H. y A. Cano (2016a). Modelos de extensión universitaria en las universidades latinoamericanas en el siglo XXI: tendencias y controversias. Universidades, LXVI(67).

Vélez Funes, C. (2020). La Escuela Campesina, expresión de la lucha política del Movimiento Campesino de Córdoba. Tesis de Doctorado. CEA. Universidad Nacional de Córdoba.

\section{Documentos}

Bowman, A.; Brumat, M. R.; Cragnolino, E. (2008). Documento de Trabajo № 1: Relevamiento de propuestas pedagógicas para la creación de una escuela secundaria en el norte de Córdoba. Demanda del Movimiento Campesino de Córdoba, Córdoba.

MCC y Secretaría de Extensión Universitaria (2008). Documento Escuela Secundaria Campesina - Córdoba. http://www.extension.unc.edu.ar/proyectoescuela- secundaria-campesina

Cragnolino, E. et al. (2009). Condiciones y alternativas de la educación rural obligatoria en el Norte de Córdoba. Documento presentado en el Foro de Educación Rural, organizado por programa de Investigaciones en Educación Rural y de Jóvenes y Adultos (CIFFY, FFYH, UNC); MCC y Programa Espacio Social de la SEUUNC, Córdoba. 